\title{
A Comparison between Adomian Decomposition and Tau Methods
}

\author{
Necdet Bildik ${ }^{1}$ and Mustafa Inc ${ }^{2}$ \\ ${ }^{1}$ Department of Mathematics, Science Faculty, Celal Bayar University, 45030 Manisa, Turkey \\ ${ }^{2}$ Department of Mathematics, Science Faculty, Firat University, 23119 Elazig, Turkey
}

Correspondence should be addressed to Necdet Bildik; necdet.bildik@cbu.edu.tr

Received 22 February 2013; Revised 7 June 2013; Accepted 8 June 2013

Academic Editor: Adem K1lıcman

Copyright (C) 2013 N. Bildik and M. Inc. This is an open access article distributed under the Creative Commons Attribution License, which permits unrestricted use, distribution, and reproduction in any medium, provided the original work is properly cited.

\begin{abstract}
We present a comparison between Adomian decomposition method (ADM) and Tau method (TM) for the integro-differential equations with the initial or the boundary conditions. The problem is solved quickly, easily, and elegantly by ADM. The numerical results on the examples are shown to validate the proposed ADM as an effective numerical method to solve the integro-differential equations. The numerical results show that ADM method is very effective and convenient for solving differential equations than Tao method.
\end{abstract}

\section{Introduction}

The decomposition method was introduced by Adomian in [1-3] in the 1980s in order to solve linear and nonlinear functional equations (algebraic, differential, partial differential equations and systems, integral, delay, integro-differential equations, etc.) [1-10]. This method leads to computable, accurate, approximate convergence solutions to linear and nonlinear deterministic and stochastic operator equations. The solution can verify any stage of approximation. The convergence of this method was proved by Cherruault and coauthors in [11-13].

In this paper we will be concerned with approximate solutions of the linear or nonlinear Volterra integro-differential equations. Firstly, this type of equation was introduced by Volterra [14] in the early 1900s. These equations can be found in physics, biology, and engineering applications such as heat transfer, diffusion process in general, and neutron diffusion [4].

Many authors have compared the ADM with some existing methods in solving different linear or nonlinear evolution equations, integral and integro-differential equations. Bellomo and Monaco [15] compared the ADM and the perturbation techniques. Advantages of the ADM over Picard's method have been shown by Rach [16]. Edwards et al. [17] compared the ADM and the Runge-Kutta methods for approximate solutions of some predator-prey models. Additionally, Wazwaz [18] presented a comparison between the ADM and the Taylor series methods. He showed that the ADM minimizes the computational difficulties of the Taylor series in that the components of the solution were determined elegantly by using simple integrals. More recently, El-Sayed and Abdel-Aziz [19] introduced a comparison of the ADM and the Wavelet-Galerkin method for the solution of integrodifferential equations. They showed that the ADM was simple and easy to use.

In [20], Hosseini and Shahmorad employed Tau method to obtain a numerical solution to the integro-differential equations given by (1). Batiha et al. [21] presented the variational iteration method (VIM) and the ADM for solving nonlinear integro-differential equations. Fariborzi Araghi and Sadigh Behzadi [22-24] solved nonlinear Volterra-Fredholm integro-differential equations by using the modified ADM, the VIM, and the homotopy analysis method, respectively. Borhanifar and Abazari [25] implemented the differential transform method for solving nonlinear integro-differential equations with the kernel functions including derivative type of unknown solution. Ben Zitoun and Cherruault [26] presented a method for solving nonlinear integro-differential equations with constant or variable coefficients with initial 
or boundary conditions. El-Kalla [27] introduced a new technique for solving a class of quadratic integral and integrodifferential equations.

In this work, we will describe and adapt Adomian's decomposition method to obtain an approximate solution for (1). As we will see, the method converges rapidly. The balance of this paper is as follows: in Section 2, we will give analysis of $\mathrm{ADM}$ for the problem; in Section 3 we will give three examples to demonstrate the method. Concluding remarks are given in the last section.

\section{Analysis}

We consider the nonlinear Volterra integro-differential equations of the form in [4] as follows:

$$
\begin{gathered}
u^{(n)}(x)=f(x)+\int_{0}^{x} K(x, t) N u(t) d t, \\
u^{(m)}(0)=c_{m}, \quad 0 \leq m \leq(n-1),
\end{gathered}
$$

where $u^{(n)}(x)$ indicates the $n$th derivative of $u(x)$ with respect to $x, c_{m}$ constants that define the initial conditions, and $N u$ is nonlinear operator. In this work we take $N u$ equal to $u^{2}$ or $u u_{x}$. Thus, applying the inverse operator $L^{-1}$ to (1) yields

$$
\begin{aligned}
u(x)= & \sum_{k=0}^{n-1} \frac{1}{k !} c_{m} x^{k}+L^{-1}[f(x)] \\
& +L^{-1}\left(\int_{0}^{x} K(x, t) N u(t) d t\right),
\end{aligned}
$$

where $\sum_{k=0}^{n-1}(1 / k !) c_{m} x^{k}$ is obtained by using the initial conditions in [4] and $L^{-1}$ is $n$-fold integration operator; that is,

$$
L^{-1}(\cdot)=\underbrace{\int_{0}^{x} \cdots \int_{0}^{x}}_{n \text {-times }}(\cdot) \underbrace{d x \cdots d x}_{n \text {-times }} .
$$

We obtain the zeroth component

$$
u_{0}(x)=\sum_{k=0}^{n-1} \frac{1}{k !} c_{m} x^{k}+L^{-1}[f(x)]
$$

which is defined by all terms that arise from the initial conditions and from integrating the source terms. Then, decomposing the unknown function $u(x)$ gives a sum of the component defined by the decomposition series

$$
u(x)=\sum_{n=0}^{\infty} u_{n}(x)
$$

Since the nonlinear terms $N u=u^{2}$ or $N u=u u_{x}$, then it can be expressed as

$$
F(u)=N u=\sum_{n=0}^{\infty} A_{n},
$$

where $A_{n}$ appropriate Adomian is polynomial which is generated form of the following formula $[1-3,6]$ :

$$
\begin{gathered}
A_{0}=F\left(u_{0}\right), \\
A_{1}=u_{1} F^{\prime}\left(u_{0}\right), \\
A_{2}=u_{2} F^{\prime}\left(u_{0}\right)+\frac{1}{2} u_{1}^{2} F^{\prime \prime}\left(u_{0}\right), \\
A_{3}=u_{3} F^{\prime}\left(u_{0}\right)+u_{1} u_{2} F^{\prime \prime}\left(u_{0}\right)+\frac{1}{3 !} u_{1}^{3} F^{\prime \prime \prime}\left(u_{0}\right),
\end{gathered}
$$

Substituting (5) and (6) into (2) yields,

$$
\begin{aligned}
\sum_{n=0}^{\infty} u_{n}(x)= & \sum_{k=0}^{n-1} \frac{1}{k !} c_{m} x^{k}+L^{-1}[f(x)] \\
& +L^{-1}\left(\int_{0}^{x} K(x, t) \sum_{n=0}^{\infty} A_{n}(t) d t\right) .
\end{aligned}
$$

The components $u_{1}(x), u_{2}(x), \ldots$ are completely determined by using the recurrent formula

$$
\begin{gathered}
u_{1}(x)=L^{-1}\left(\int_{0}^{x} K(x, t) A_{0}(t) d t\right), \\
\vdots \\
u_{n}(x)=L^{-1}\left(\int_{0}^{x} K(x, t) A_{n-1}(t) d t\right),
\end{gathered}
$$

for $n \geq 0$. It is useful to note that the recursive formula is constructed on the basis that the zeroth component $u_{0}(x)$ is defined by all terms that arise from the initial conditions and from integrating the source terms. The remaining components $u_{n}(x), n \geq 0$, can be completely determined such that each term is computed by using the previous term. As a result, the components $u_{0}(x), u_{1}(x), u_{2}(x), \ldots$ are identified, and the series solutions are thus entirely determined.

The $n$-term approximation $\phi_{n}$ is defined by

$$
\phi_{n}=\sum_{k=0}^{n-1} u_{k}(x) \text {, }
$$

which can be used for numerical approximation.

\section{Test Problems}

In this section, we report on numerical results of some examples, selected through integral and integro-differential equations, solved by ADM. These examples can be solved analytically by reducing them to differential equations, and they are also solved numerically by Tau method in [20]. Here the aim is to solve these examples using the ADM given Section 2 and compare these results with the presented results in [20]. 
Problem 1 . We mainly present the method using the algorithm given in Section 2. As a first example, consider the equation

$$
y(s)-\int_{0}^{s} y(t) d t=1, \quad 0 \leq s \leq 1
$$

In order to illustrate the proposed method, we get zeroth component

$$
y_{0}=1
$$

and obtain $y_{1}(x), y_{2}(x), \ldots$ by using (9) to determine the other individual terms of the decomposition series. Thus

$$
\begin{gathered}
y_{1}=\int_{0}^{s} y_{0}(t) d t=s, \\
y_{2}=\int_{0}^{s} y_{1}(t) d t=\frac{1}{2 !} s^{2}, \\
y_{3}=\int_{0}^{s} y_{2}(t) d t=\frac{1}{3 !} s^{3},
\end{gathered}
$$

and so on. Consequently, the series solution is obtained as

$$
\begin{aligned}
y(s) & =\sum_{n=0}^{\infty} y_{n}(s)=y_{0}+y_{1}+y_{2}+y_{3}+\cdots \\
& =1+s+\frac{1}{2 !} s^{2}+\frac{1}{3 !} s^{3}+\cdots,
\end{aligned}
$$

so that the closed form of the solution is

$$
y(s)=e^{s}
$$

Problem 2. We consider Fredholm integro-differential equation which is given as follows [20]:

$$
\begin{aligned}
& y^{\prime \prime}(s)-y(s)+\frac{1}{20} \int_{0}^{1} t^{39} y(t) d t \\
& =-s^{2}-2 s+\frac{2111}{344400}, \quad 0 \leq s \leq 1, \\
& y(0)-y^{\prime}(0)=0, \quad y(1)+y^{\prime}(1)=9 .
\end{aligned}
$$

Proceeding as before, we obtain

$$
\begin{aligned}
y_{0}= & y(0)+s y^{\prime}(0)+L^{-1}\left(-s^{2}-2 s+\frac{2111}{344400}\right) \\
= & 2+2 s+\frac{2111}{688800} s^{2}-\frac{1}{3} s^{3}-\frac{1}{12} s^{4} \\
y_{1}= & \int_{0}^{s} \int_{0}^{s}\left(y_{0}(s)\right) d s d s \\
& -\frac{1}{20} \int_{0}^{s} \int_{0}^{s}\left(\int_{0}^{1} t^{39} y_{0}(t) d t\right) d s d s \\
= & s^{2}-\frac{29772923}{13349952000} s^{2}+\frac{1}{3} s^{3}+\frac{2111}{8265600} s^{4} \\
y_{2}= & \int_{0}^{s} \int_{0}^{s}\left(y_{1}(s)\right) d s d s \\
& -\frac{1}{60} s_{0}^{5}-\frac{1}{360} s^{5}, \int_{0}^{s}\left(\int_{0}^{139} y_{1}(t) d t\right) d s d s \\
= & \frac{-5478328678327}{7049842652160000} s^{2}+\frac{13320179077}{160199424000} s^{4} \\
+ & \frac{1}{60} s^{5}+\frac{2111}{247968000} s^{6}-\frac{1}{2520} s^{7}-\frac{1}{201160} s^{8},
\end{aligned}
$$

Consequently, the series solution is found as

$$
\begin{aligned}
y(x)= & \sum_{n=0}^{\infty} y_{n}(x)=y_{0}+y_{1}+y_{2}+\cdots \\
= & 2+2 s+\frac{2111}{688800} s^{2}-\frac{1}{3} s^{3}-\frac{1}{12} s^{4} \\
& +s^{2}-\frac{29772923}{13349952000} s^{2}+\frac{1}{3} s^{3}+\cdots .
\end{aligned}
$$

In Table 1, ADM and TM values are presented which correspond to the various values of $s$. As it is seen in this table, the values obtained by [20] and the results we obtained which are close and but present method better accuracy and easy to use than the TM. It is to be noted that only few iterations were needed to obtain the accuracy for approximate solutions. The overall errors can be made even much smaller by adding new terms of the decomposition. Thus the convergence would be seen more rapidly.

The numerical solutions showed that ADM is a very convenient method for such linear and nonlinear integral and integro-differential equations. By using this method, it is possible to obtain more precise results than the traditional methods, with less calculations and consuming the less time.

Problem 3. In $[4,28]$, Wazwaz proposed that the construction of the zeroth component of the decomposition series can be defined in a slightly different way. In $[4,28]$, he assumed that if 
TABLe 1: Numerical results for Problem 2.

\begin{tabular}{llllcc}
\hline$s$ & Exact & Tau $(n)$ & ADM $(\phi)$ & Tau-Er. & ADM-Er. \\
\hline \multicolumn{5}{c}{$n=2, \phi_{2}$} \\
0.00 & 2.00000 & 1.99878 & 2.00000 & $1.2200 e-03$ & $0.000 e+00$ \\
0.20 & 2.44000 & 2.43858 & 2.43989 & $1.4152 e-03$ & $1.051 e-04$ \\
0.40 & 2.96000 & 2.95849 & 2.95782 & $1.5128 e-03$ & $2.175 e-03$ \\
0.60 & 3.56000 & 3.55849 & 3.54811 & $1.5128 e-03$ & $1.200 e-02$ \\
\hline \multicolumn{5}{c}{$n=5, \phi_{3}$} \\
0.00 & 2.00000 & 1.99885 & 2.00000 & $1.1525 e-03$ & $0.000 e+00$ \\
0.20 & 2.44000 & 2.43867 & 2.44000 & $1.3342 e-03$ & $2.228 e-06$ \\
0.40 & 2.96000 & 2.95858 & 2.95999 & $1.4226 e-03$ & $1.049 e-06$ \\
0.60 & 3.56000 & 3.55858 & 3.55988 & $1.4211 e-03$ & $1.114 e-04$ \\
0.80 & 4.24000 & 4.23867 & 4.23924 & $1.3308 e-03$ & $7.522 e-04$ \\
1.00 & 5.00000 & 4.99885 & 4.99691 & $1.1485 e-03$ & $3.089 e-03$ \\
\hline & & & $n=15, \phi_{5}$ & & \\
0.00 & 2.00000 & 1.99884 & 2.00000 & $1.1567 e-03$ & $0.000 e+00$ \\
0.20 & 2.44000 & 2.43866 & 2.44000 & $1.3394 e-03$ & $6.597 e-08$ \\
0.40 & 2.96000 & 2.95857 & 2.96000 & $1.4289 e-03$ & $2.671 e-07$ \\
0.60 & 3.56000 & 3.55857 & 3.56000 & $1.4288 e-03$ & $6.143 e-07$ \\
0.80 & 4.24000 & 4.23866 & 4.24000 & $1.3394 e-03$ & $1.940 e-06$ \\
1.00 & 5.00000 & 4.99884 & 5.00000 & $1.1567 e-03$ & $1.380 e-06$ \\
\hline
\end{tabular}

the zeroth component is $y_{0}=f$ and the function $f$ is possible to be divided into two parts such as $f_{1}$ and $f_{2}$, then one can formulate the recursive algorithm in a form of a modified recursive scheme as follows:

$$
\begin{gathered}
y_{0}(s)=f_{1} \\
y_{1}(s)=f_{2}+L^{-1}[f(x)]+L^{-1}\left(\int_{0}^{x} K(x, t) y_{1}(t) d t\right), \\
y_{n+1}(s)=L^{-1}\left(\int_{0}^{x} K(x, t) y_{n}(t) d t\right), \quad n \geq 1 .
\end{gathered}
$$

We finally consider the Volterra integral equation in the following form [20]:

$$
\begin{aligned}
y(s)=1 & +120 s-100\left(1-e^{-s}\right) \\
& +\int_{0}^{s}\left(100 e^{t-s}-120\right) y(t) d t, \quad 0 \leq s \leq 20 .
\end{aligned}
$$

Using the modified decomposition method, we first decompose the function $f(s)$ into two parts as $f_{1}$ and $f_{2}$, namely,

$$
\begin{gathered}
f_{1}(s)=1 \\
f_{2}(s)=120 s-100\left(1-e^{-s}\right) .
\end{gathered}
$$

Consequently, we obtain

$$
\begin{gathered}
y_{0}(s)=1, \\
y_{1}(s)=120 s-100\left(1-e^{-s}\right) \\
+\int_{0}^{s}\left(100 e^{t-s}-120\right) y_{0}(t) d t=0 .
\end{gathered}
$$

Other components $y_{n}(s)=0$ for $n \geq 2$. Therefore, the exact solution

$$
y(s)=1
$$

follows immediately. It is clear that two components are calculated to determine the exact solution.

\section{Concluding Remarks}

In this paper, we calculated the approximate solutions of the integral and Volterra integro-differential equations by using Adomian decomposition method. We demonstrated that the decomposition procedure is quite efficient in order to determine the solution in closed form by using initial and boundary conditions. Our present method avoids the tedious work needed by traditional techniques. In the studies by Hosseini and Shahmorad in [20], they spent more time, and boring operations were done to get approximate solutions by using TM. In our study, however, we got more accurate approximate solutions by using the initial condition in this method; Hosseini and Shahmorad in [20] obtained the approximate solutions for Problems 1 and 3, such that, the Tau-error is $2.73127 e-08$ for $n=10$ and $s=1.00$ in Problem 1. Moreover, the exact solutions are obtained by our present method for Problems 1 and 3. Our method avoids the difficulties and massive computational work that usually arise from Wavelet-Galerkin, Tau, and finite difference methods.

\section{References}

[1] G. Adomian, Nonlinear Stochastic Operator Equations, Academic Press, Orlando, Fla, USA, 1986.

[2] G. Adomian, Nonlinear Stochastic Systems and Application to Physics, Kluwer Academic Publishers, Norwell, Mass, USA, 1989.

[3] G. Adomian, Solving Frontier Problems of Physics: The Decomposition Method, vol. 60 of Fundamental Theories of Physics, Kluwer Academic Publishers, Dordrecht, The Netherlands, 1994.

[4] A.-M. Wazwaz, A First Course in Integral Equations, World Scientific Publishing, River Edge, NJ, USA, 1997.

[5] M. Inc and Y. Cherruault, "A new approach to solve a diffusionconvection problem," Kybernetes, vol. 31, no. 3-4, pp. 354-355, 536-549, 2002.

[6] A.-M. Wazwaz, "A new algorithm for calculating Adomian polynomials for nonlinear operators," Applied Mathematics and Computation, vol. 111, no. 1, pp. 53-69, 2000.

[7] M. Inc and Y. Cherruault, "A new approach to travelling wave solution of a fourth-order semilinear diffusion equation," Kybernetes, vol. 32, pp. 1492-1503, 2003.

[8] M. Inc, Y. Cherruault, and K. Abbaoui, "A computational approach to the wave equations: an application of the decomposition method," Kybernetes, vol. 33, no. 1, pp. 80-97, 2004.

[9] E. Deeba, S. A. Khuri, and S. Xie, "An algorithm for solving a nonlinear integro-differential equation," Applied Mathematics and Computation, vol. 115, no. 2-3, pp. 123-131, 2000.

[10] M. Inc and D. J. Evans, "A different approach for soliton solution of the improved Boussinesq equation," International Journal of Computer Mathematics, vol. 81, no. 3, pp. 313-323, 2004. 
[11] Y. Cherruault, "Convergence of Adomian's method," Kybernetes, vol. 18, no. 2, pp. 31-38, 1989.

[12] Y. Cherruault, G. Saccomandi, and B. Some, "New results for convergence of Adomian's method applied to integral equations," Mathematical and Computer Modelling, vol. 16, no. 2, pp. 85-93, 1992.

[13] Y. Cherruault and G. Adomian, "Decomposition methods: a new proof of convergence," Mathematical and Computer Modelling, vol. 18, no. 12, pp. 103-106, 1993.

[14] V. Volterra, Theory of Functionals and of Integral and IntegroDifferential Equations, Dover, New York, NY, USA, 1959.

[15] N. Bellomo and R. Monaco, “A comparison between Adomian's decomposition methods and perturbation techniques for nonlinear random differential equations," Journal of Mathematical Analysis and Applications, vol. 110, no. 2, pp. 495-502, 1985.

[16] R. Rach, "On the Adomian (decomposition) method and comparisons with Picard's method," Journal of Mathematical Analysis and Applications, vol. 128, no. 2, pp. 480-483, 1987.

[17] J. Y. Edwards, J. A. Roberts, and N. J. Ford, "A comparison of Adomian's decomposition method and Runge-Kutta methods for approximate solution of some predator prey model equations," Tech. Rep. 309, Manchester Center of Computational Mathematics, Manchester, UK, 1997.

[18] A.-M. Wazwaz, "A comparison between Adomian decomposition method and Taylor series method in the series solutions," Applied Mathematics and Computation, vol. 97, no. 1, pp. 37-44, 1998.

[19] S. M. El-Sayed and M. R. Abdel-Aziz, "A comparison of Adomian's decomposition method and wavelet-Galerkin method for solving integro-differential equations," Applied Mathematics and Computation, vol. 136, no. 1, pp. 151-159, 2003.

[20] S. M. Hosseini and S. Shahmorad, "Numerical solution of a class of integro-differential equations by the tau method with an error estimation," Applied Mathematics and Computation, vol. 136, no. 2-3, pp. 559-570, 2003.

[21] B. Batiha, M. S. M. Noorani, and I. Hashim, "Numerical solutions of the nonlinear integro-differential equations," International Journal of Open Problems in Computer Science and Mathematics, vol. 1, no. 1, pp. 34-42, 2008.

[22] M. A. Fariborzi Araghi and Sh. Sadigh Behzadi, "Solving nonlinear Volterra-Fredholm integro-differential equations using the modified Adomian decomposition method," Computational Methods in Applied Mathematics, vol. 9, no. 4, pp. 321-331, 2009.

[23] M. A. Fariborzi Araghi and Sh. Sadigh Behzadi, "Solving nonlinear Volterra-Fredholm integro-differential equations using He's variational iteration method," International Journal of Computer Mathematics, vol. 88, no. 4, pp. 829-838, 2011.

[24] M. A. Fariborzi Araghi and S. S. Behzadi, "Numerical solution of nonlinear Volterra-Fredholm integro-differential equations using homotopy analysis method," Journal of Applied Mathematics and Computing, vol. 37, no. 1-2, pp. 1-12, 2011.

[25] A. Borhanifar and R. Abazari, "Differential transform method for a class of nonlinear integro-differential equations with derivative type kernel," Canadian Journal on Computing in Mathematics, vol. 3, pp. 1-6, 2012.

[26] F. Ben Zitoun and Y. Cherruault, "A method for solving nonlinear integro-differential equations," Kybernetes, vol. 41, no. 1-2, pp. 35-50, 2012.

[27] I. L. El-Kalla, "A new approach for solving a class of nonlinear integro-differential equations," Communications in Nonlinear Science and Numerical Simulation, vol. 17, no. 12, pp. 4634-4641, 2012.
[28] A.-M. Wazwaz, "A reliable modification of Adomian decomposition method," Applied Mathematics and Computation, vol. 102, no. 1, pp. 77-86, 1999. 


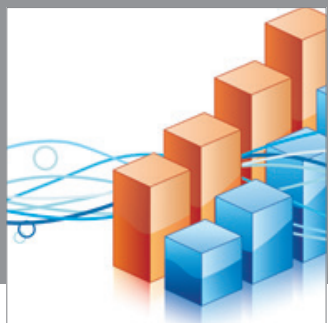

Advances in

Operations Research

mansans

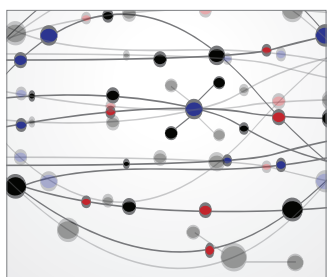

The Scientific World Journal
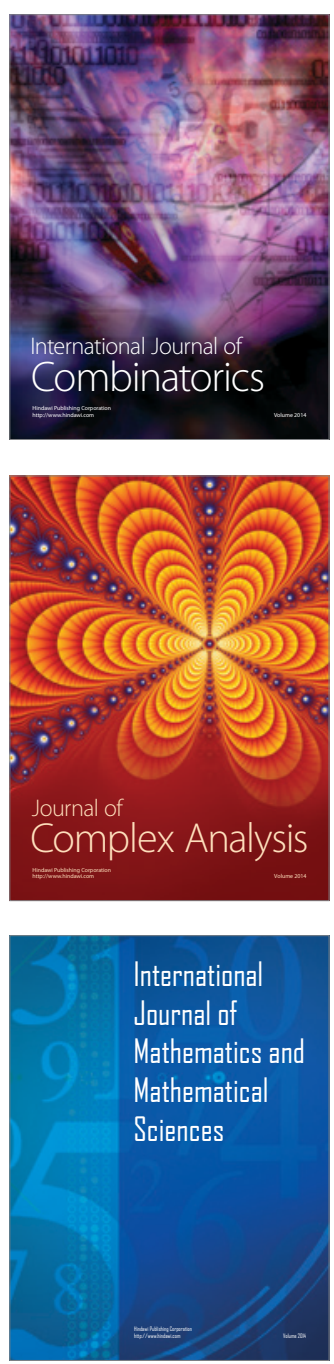
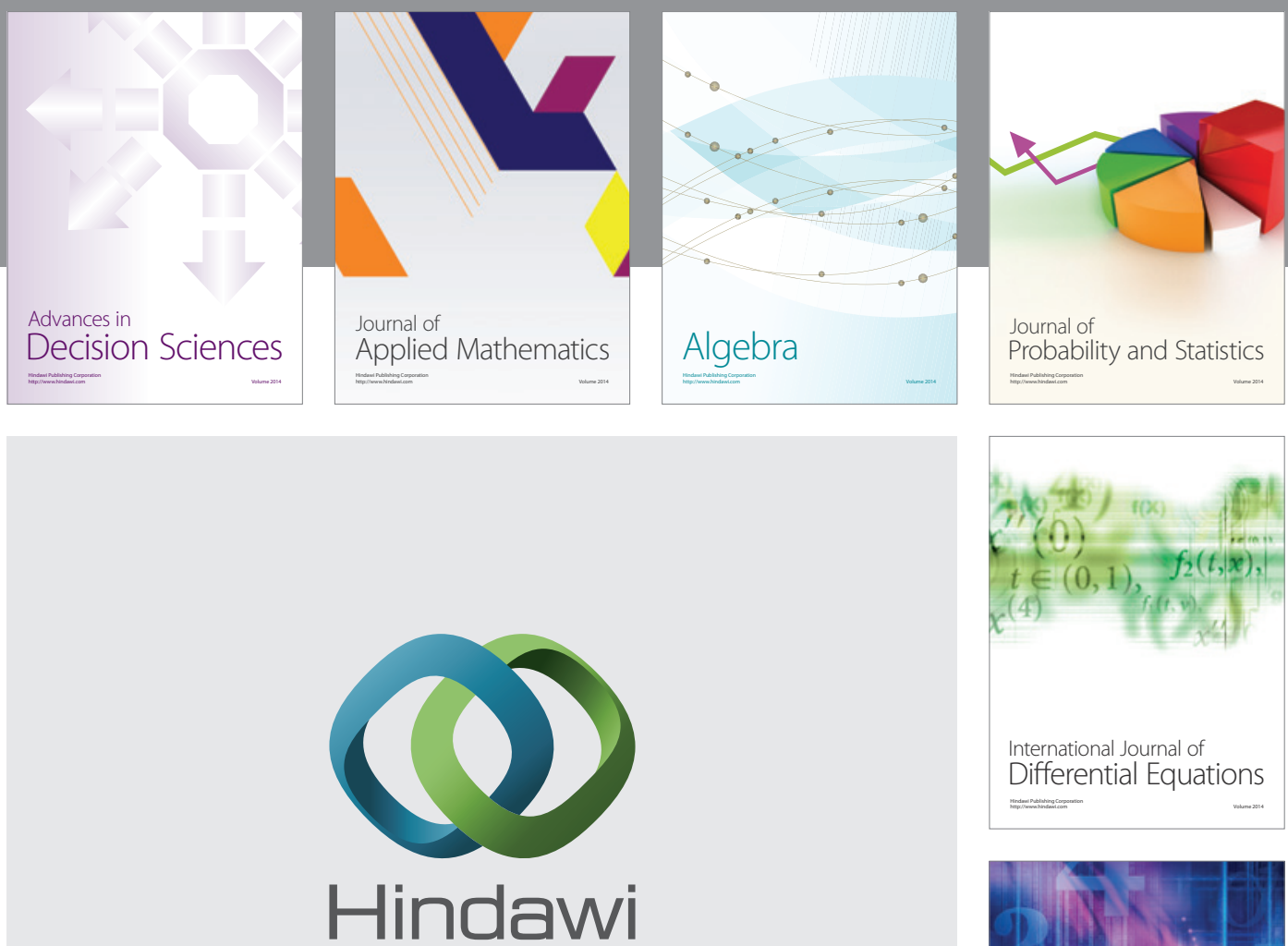

Submit your manuscripts at http://www.hindawi.com
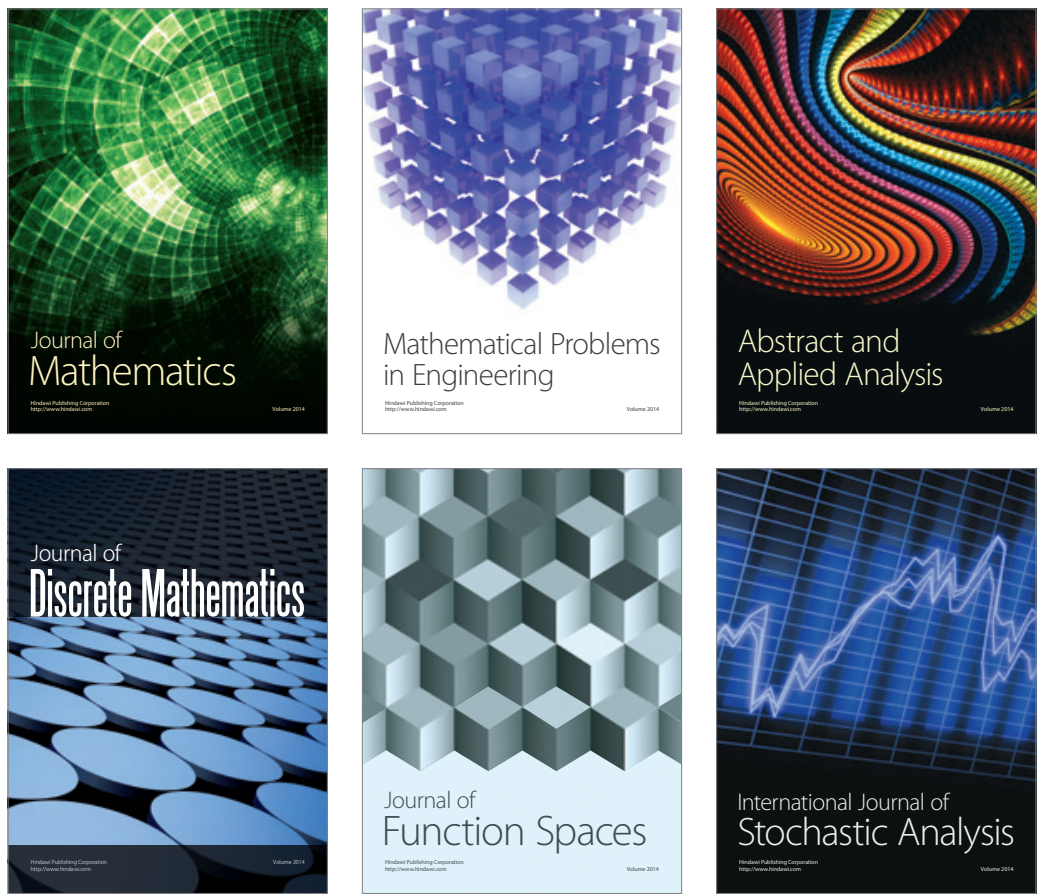

Journal of

Function Spaces

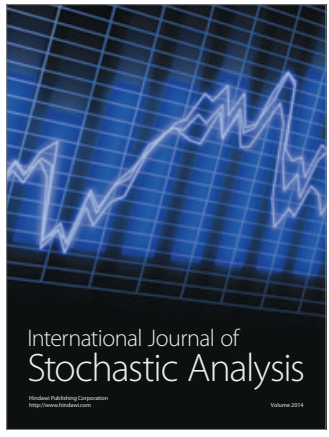

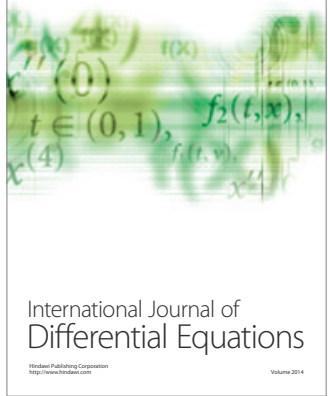
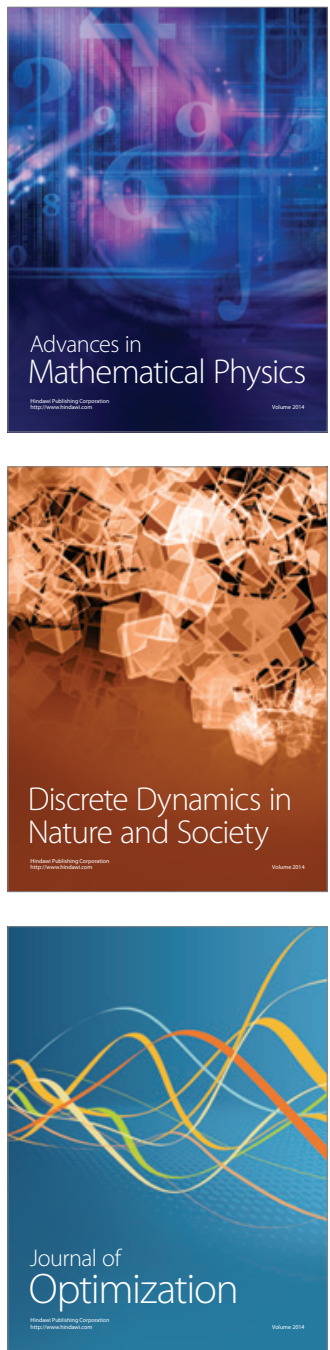